\title{
Scaled Dimension and Nonuniform Complexity
}

\author{
John M. Hitchcock * \\ Department of Computer Science \\ University of Wyoming \\ Laramie, WY 82071, USA \\ jhitchco@cs.uwyo.edu
}

\author{
Jack H. Lutz ${ }^{\dagger}$ \\ Department of Computer Science \\ Iowa State University \\ Ames, IA 50011, USA \\ lutz@cs.iastate.edu
}

Elvira Mayordomo $\ddagger$

Departamento de Informática e Ingeniería de Sistemas

Universidad de Zaragoza

50015 Zaragoza, SPAIN

elvira@posta.unizar.es

\begin{abstract}
Resource-bounded dimension is a complexity-theoretic extension of classical Hausdorff dimension introduced by Lutz (2000) in order to investigate the fractal structure of sets that have resource-bounded measure 0 . For example, while it has long been known that the Boolean circuit-size complexity class $\operatorname{SIZE}\left(\alpha \frac{2^{n}}{n}\right)$ has measure 0 in ESPACE for all $0 \leq \alpha \leq 1$, we now know that $\operatorname{SIZE}\left(\alpha \frac{2^{n}}{n}\right)$ has dimension $\alpha$ in ESPACE for all $0 \leq \alpha \leq 1$.

The present paper furthers this program by developing a natural hierarchy of "rescaled" resource-bounded dimensions. For each integer $i$ and each set $X$ of decision problems, we define the $i^{\text {th }}$-order dimension of $X$ in suitable complexity classes. The $0^{\text {th }}$-order dimension is precisely the dimension of Hausdorff (1919) and Lutz (2000). Higher and lower orders are useful for various sets $X$. For example, we prove the following for $0 \leq \alpha \leq 1$ and any polynomial $q(n) \geq n^{2}$.

1. The class $\operatorname{SIZE}\left(2^{\alpha n}\right)$ and the time- and space-bounded Kolmogorov complexity classes $\mathrm{KT}^{q}\left(2^{\alpha n}\right)$ and $\mathrm{KS}^{q}\left(2^{\alpha n}\right)$ have $1^{\text {st }}$-order dimension $\alpha$ in ESPACE.

2. The classes $\operatorname{SIZE}\left(2^{n^{\alpha}}\right), \mathrm{KT}^{q}\left(2^{n^{\alpha}}\right)$, and $\mathrm{KS}^{q}\left(2^{n^{\alpha}}\right)$ have $2^{\text {nd }}$-order dimension $\alpha$ in ESPACE.

3. The classes $\mathrm{KT}^{q}\left(2^{n}\left(1-2^{-\alpha n}\right)\right)$ and $\mathrm{KS}^{q}\left(2^{n}\left(1-2^{-\alpha n}\right)\right.$ have $-1^{\text {st }}$-order dimension $\alpha$ in ESPACE.
\end{abstract}

\section{Introduction}

Many sets of interest in computational complexity have quantitative structures that are too fine to be elucidated by resource-bounded measure. For example, it has long been known that the Boolean circuit-size complexity class $\operatorname{SIZE}\left(\frac{2^{n}}{n}\right)$ has measure 0 in ESPACE [13], so resource-bounded measure cannot make quantitative distinctions among subclasses of $\operatorname{SIZE}\left(\frac{2^{n}}{n}\right)$.

\footnotetext{
*This research was supported in part by National Science Foundation Grant 9988483.

${ }^{\dagger}$ This research was supported in part by National Science Foundation Grants 9610461 and 9988483.

‡This research was supported in part by Spanish Government projects PB98-0937-C04-02 and TIC2002-04019-C03. It was done while visiting Iowa State University.
} 
In early 2000, Lutz [14] developed resource-bounded dimension in order to remedy this situation. Just as resource-bounded measure is a complexity-theoretic generalization of classical Lebesgue measure, resource-bounded dimension is a complexity-theoretic generalization of classical Hausdorff dimension. Moreover, just as classical Hausdorff dimension enables us to quantify the structures of many sets of Lebesgue measure 0, resource-bounded dimension enables us to quantify the structures of some sets that have measure 0 in complexity classes. For example, Lutz [14] showed that for every real number $\alpha \in[0,1]$, the class $\operatorname{SIZE}\left(\alpha \frac{2^{n}}{n}\right)$ has dimension $\alpha$ in ESPACE. He also showed that for every p-computable $\alpha \in[0,1]$, the class of languages with limiting frequency $\alpha$ has dimension $\mathcal{H}(\alpha)$ in E, where $\mathcal{H}$ is the binary entropy function of Shannon information theory. (This is a complexity-theoretic extension of a classical result of Eggleston [4].) These preliminary results suggest new relationships between information and complexity and open the way for investigating the fractal structure of complexity classes. More recent work has already used resource-bounded dimension to illuminate a variety of topics in computational complexity $[1,2,6,8,9,3]$.

However, there is a conspicuous obstacle to further progress along these lines. Many classes that occur naturally in computational complexity are parametrized in such a way as to remain out of reach of the resource-bounded dimension of [14]. For example, when discussing cryptographic security or derandomization, one is typically interested in circuit-size bounds of the form $2^{\alpha n}$ or $2^{n^{\alpha}}$, rather than the $\alpha \frac{2^{n}}{n}$ bound of the above-cited result. It is easy to see that for all $\alpha<1$, $\operatorname{SIZE}\left(2^{\alpha n}\right)$ and $\operatorname{SIZE}\left(2^{n^{\alpha}}\right)$ have dimension 0 in ESPACE, so the resource-bounded dimension of [14] cannot provide the sort of quantitative classification that is needed. Similarly, in their investigations of the information content of complete problems, Juedes and Lutz [10] established tight bounds on space-bounded Kolmogorov complexity of the forms $2^{n^{\epsilon}}$ and $2^{n+1}-2^{n^{\epsilon}}$; in the investigation of completeness in $\mathrm{E}$ one is typically interested in dense languages, which have census at least $2^{n^{\epsilon}}$; etc. The difficulty here is that classes arising naturally in computational complexity are often scaled in a nonlinear way that is not compatible with the linear scaling implicit in classical Hausdorff dimension and the resource-bounded dimension of Lutz [14].

This sort of difficulty has already been encountered in the classical theory of Hausdorff dimension and dealt with by rescaling the dimension. The 1970 classic [17] by C.A. Rogers describes the resulting theory of generalized dimension, in which Hausdorff dimension may be rescaled by any element of a very large class of extended real-valued functions. (In fact, this idea was introduced in Hausdorff's 1919 paper [7].) Choosing the right such function for a particular set often yields more precise information about that set's dimension. For example, it is known that with probability 1 a Brownian sample path in the plane has Hausdorff dimension 2 (the dimension of the plane), but a more careful analysis using the generalized approach shows that "the dimension is, in a sense, logarithmically smaller than 2" [5].

In this paper we extend the resource-bounded dimension of [14] by introducing the notion of a scale according to which dimension may be measured. Our scales are slightly less general than the functions used for generalized dimension and take two arguments instead of one, but every scale $g$ defines for every set $X$ of decision problems a $g$-scaled dimension $\operatorname{dim}^{(g)}(X) \in[0,1]$. Thus, although the spirit of our approach is much like that of generalized dimension, scaled dimension typically yields quantitative results that are as precise as, but crisper than, the result quoted at the end of the preceding paragraph.

The choice of which scale to use for a particular application is very much like the choice of whether to plot data on a standard Cartesian graph or a log-log graph. In fact, a very restricted family of scales appears to be adequate for analyzing many problems in computational complexity. Specifically, we define a particular, natural hierarchy of scales, one for each integer, and use these to define the $i^{\text {th }}$-order dimension of arbitrary sets $X$ in suitable complexity classes. The $0^{\text {th }}$-order 
dimension is precisely the dimension used by Hausdorff [7] and Lutz [14]. We propose that higherand lower-order dimensions will be useful for many investigations in computational complexity. In support of this proposal we prove the following for $0 \leq \alpha \leq 1$ and any polynomial $q(n) \geq n^{2}$.

1. The class $\operatorname{SIZE}\left(2^{\alpha n}\right)$ and the time- and space-bounded Kolmogorov complexity classes $\operatorname{KT}^{q}\left(2^{\alpha n}\right)$ and $\operatorname{KS}^{q}\left(2^{\alpha n}\right)$ have $1^{\text {st }}$-order dimension $\alpha$ in ESPACE.

2. The classes $\operatorname{SIZE}\left(2^{n^{\alpha}}\right), \mathrm{KT}^{q}\left(2^{n^{\alpha}}\right)$, and $\mathrm{KS}^{q}\left(2^{n^{\alpha}}\right)$ have $2^{\text {nd }}$-order dimension $\alpha$ in ESPACE.

3. The classes $\mathrm{KT}^{q}\left(2^{n}\left(1-2^{-\alpha n}\right)\right)$ and $\mathrm{KS}^{q}\left(2^{n}\left(1-2^{-\alpha n}\right)\right.$ have $-1^{\text {st }}$-order dimension $\alpha$ in ESPACE.

We emphasize that, for all $\alpha \in(0,1)$, all these classes have measure 0 in ESPACE, the classes in 1 and 2 have $0^{\text {th }}$-order dimension 0 in ESPACE, and the class in 3 has $0^{\text {th }}$-order dimension 1 in ESPACE. Only when the dimension is appropriately rescaled does it respond informatively to variation of the parameter $\alpha$.

We also prove more general results along these lines.

\section{Preliminaries}

A decision problem (a.k.a. language) is a set $A \subseteq\{0,1\}^{*}$. We identify each language with its characteristic sequence $\llbracket s_{0} \in A \rrbracket \llbracket s_{1} \in A \rrbracket \llbracket s_{2} \in A \rrbracket \cdots$, where $s_{0}, s_{1}, s_{2}, \ldots$ is the standard enumeration of $\{0,1\}^{*}$ and

$$
\llbracket \phi \rrbracket=\text { if } \phi \text { then } 1 \text { else } 0 .
$$

We write $A[i . . j]$ for the string consisting of the $i$-th through the $j$-th bits of (the characteristic sequence of) $A$. The Cantor space $\mathbf{C}$ is the set of all decision problems.

If $w \in\{0,1\}^{*}$ and $x \in\{0,1\}^{*} \cup \mathbf{C}$, then $w \sqsubseteq x$ means that $w$ is a prefix of $x$. The cylinder generated by a string $w \in\{0,1\}^{*}$ is $\mathbf{C}_{w}=\{A \in \mathbf{C} \mid w \sqsubseteq A\}$.

A prefix set is a language $A$ such that no element of $A$ is a prefix of any other element of $A$.

If $A$ is a language and $n \in \mathbb{N}$, then we write $A_{=n}=A \cap\{0,1\}^{n}, A_{\leq n}=A \cap\{0,1\} \leq n$.

All logarithms in this paper are base 2 .

For each $i \in \mathbb{N}$ we define a class $G_{i}$ of functions from $\mathbb{N}$ into $\mathbb{N}$ as follows.

$$
\begin{aligned}
G_{0} & =\left\{f \mid(\exists k)\left(\forall^{\infty} n\right) f(n) \leq k n\right\} \\
G_{i+1} & =2^{G_{i}(\log n)}=\left\{f \mid\left(\exists g \in G_{i}\right)\left(\forall^{\infty} n\right) f(n) \leq 2^{g(\log n)}\right\}
\end{aligned}
$$

We also define the functions $\hat{g}_{i} \in G_{i}$ by $\hat{g}_{0}(n)=2 n, \hat{g}_{i+1}(n)=2^{\hat{g}_{i}(\log n)}$. We regard the functions in these classes as growth rates. In particular, $G_{0}$ contains the linearly bounded growth rates and $G_{1}$ contains the polynomially bounded growth rates. It is easy to show that each $G_{i}$ is closed under composition, that each $f \in G_{i}$ is $o\left(\hat{g}_{i+1}\right)$, and that each $\hat{g}_{i}$ is $o\left(2^{n}\right)$. Thus $G_{i}$ contains superpolynomial growth rates for all $i>1$, but all growth rates in the $G_{i}$-hierarchy are subexponential.

Within the class of all decidable languages, we are interested in the exponential complexity classes $\mathrm{E}_{i}=\operatorname{DTIME}\left(2^{G_{i-1}}\right)$ and $\mathrm{E}_{i} \mathrm{SPACE}=\operatorname{DSPACE}\left(2^{G_{i-1}}\right)$ for $i \geq 1$. The much-studied classes $\mathrm{E}=\mathrm{E}_{1}=\operatorname{DTIME}\left(2^{\text {linear }}\right), \mathrm{E}_{2}=\operatorname{DTIME}\left(2^{\text {polynomial }}\right)$, and $\operatorname{ESPACE}=\mathrm{E}_{1} \mathrm{SPACE}=$ $\operatorname{DSPACE}\left(2^{\text {linear }}\right)$ are of particular interest.

We use the following classes of functions.

all $=\left\{f \mid f:\{0,1\}^{*} \rightarrow\{0,1\}^{*}\right\}$

rec $=\{f \in$ all $\mid f$ is computable $\}$

$\mathrm{p}_{i}=\left\{f \in\right.$ all $\mid f$ is computable in $G_{i}$ time $\}(i \geq 1)$ 
$\mathrm{p}_{i}$ space $=\left\{f \in\right.$ all $\mid f$ is computable in $G_{i}$ space $\}(i \geq 1)$

(The length of the output is included as part of the space used in computing $f$.) We write p for $\mathrm{p}_{1}$ and pspace for $\mathrm{p}_{1}$ space. Throughout this paper, $\Delta$ and $\Delta^{\prime}$ denote one of the classes all, rec, $\mathrm{p}_{i}(i \geq 1), \mathrm{p}_{i}$ space $(i \geq 1)$.

A constructor is a function $\delta:\{0,1\}^{*} \rightarrow\{0,1\}^{*}$ that satisfies $x \neq \delta(x)$ for all $x$. The result of a constructor $\delta$ (i.e., the language constructed by $\delta$ ) is the unique language $R(\delta)$ such that $\delta^{n}(\lambda) \sqsubseteq R(\delta)$ for all $n \in \mathbb{N}$. Intuitively, $\delta$ constructs $R(\delta)$ by starting with $\lambda$ and then iteratively generating successively longer prefixes of $R(\delta)$. We write $R(\Delta)$ for the set of languages $R(\delta)$ such that $\delta$ is a constructor in $\Delta$. The following facts are the reason for our interest in the above-defined classes of functions.

$$
\begin{aligned}
& R(\text { all })=\mathbf{C} \text {. } \\
& R(\mathrm{rec})=\mathrm{REC} \text {. } \\
& \text { For } i \geq 1, R\left(\mathrm{p}_{i}\right)=\mathrm{E}_{i} \text {. } \\
& \text { For } i \geq 1, R\left(\mathrm{p}_{i} \text { space }\right)=\mathrm{E}_{i} \mathrm{SPACE} \text {. }
\end{aligned}
$$

If $D$ is a discrete domain, then a function $f: D \longrightarrow[0, \infty)$ is $\Delta$-computable if there is a function $\hat{f}: \mathbb{N} \times D \longrightarrow \mathbb{Q} \cap[0, \infty)$ such that $|\hat{f}(r, x)-f(x)| \leq 2^{-r}$ for all $r \in \mathbb{N}$ and $x \in D$ and $\hat{f} \in \Delta$ (with $r$ coded in unary and the output coded in binary). We say that $f$ is exactly $\Delta$-computable if $f: D \longrightarrow \mathbb{Q} \cap[0, \infty)$ and $f \in \Delta$.

\section{Scaled Dimension}

In this section we develop a theory of scaled dimensions in complexity classes. We then develop a particular, natural hierarchy of scaled dimensions that are suitable for complexity-theoretic applications such as those in section 4.

Definition. A scale is a continuous function $g: H \times[0, \infty) \longrightarrow \mathbb{R}$ with the following properties.

1. $H=(a, \infty)$ for some $a \in \mathbb{R} \cup\{-\infty\}$.

2. $g(m, 1)=m$ for all $m \in H$.

3. $g(m, 0)=g\left(m^{\prime}, 0\right) \geq 0$ for all $m, m^{\prime} \in H$.

4. For every sufficiently large $m \in H$, the function $s \mapsto g(m, s)$ is nonnegative and strictly increasing.

5. For all $s^{\prime}>s \geq 0, \lim _{m \rightarrow \infty}\left[g\left(m, s^{\prime}\right)-g(m, s)\right]=\infty$.

Example 3.1. The function $g_{0}: \mathbb{R} \times[0, \infty) \rightarrow \mathbb{R}$ defined by $g_{0}(m, s)=s m$ is the canonical example of a scale.

Example 3.2. The function $g_{1}:(0, \infty) \times[0, \infty) \rightarrow \mathbb{R}$ defined by $g_{1}(m, s)=m^{s}$ is also a scale.

Definition. If $g: H \times[0, \infty) \rightarrow \mathbb{R}$ is a scale, then the first rescaling of $g$ is the function $g^{\#}$ : $H^{\#} \times[0, \infty) \longrightarrow \mathbb{R}$ defined by

$$
\begin{gathered}
H^{\#}=\left\{2^{m} \mid m \in H\right\} \\
g^{\#}(m, s)=2^{g(\log m, s)} .
\end{gathered}
$$


Note that $g_{0}^{\#}=g_{1}$, where $g_{0}$ and $g_{1}$ are the scales of Examples 3.1 and 3.2.

If $g$ is a scale, then for all $m \in H^{\#}$ and $s \in[0, \infty)$,

$$
\log g^{\#}(m, s)=g(\log m, s),
$$

which means that a log-log graph of the function $m \mapsto g^{\#}(m, s)$ is precisely the ordinary graph of the function $m \mapsto g(m, s)$. This is the sense in which $g^{\#}$ is a rescaling of $g$.

Lemma 3.3. If $g$ is a scale, then $g^{\#}$ is a scale.

Proof. Let $g: H \times[0, \infty) \rightarrow \mathbb{R}$ be a scale, where $H=(a, \infty)$.

1. It is clear that $H^{\#}=\left(2^{a}, \infty\right)$.

2. For $m \in H^{\#}$ we have $\log m \in H$, so $g^{\#}(m, 1)=2^{g(\log m, 1)}=2^{\log m}=m$.

3. If $m, m^{\prime} \in H^{\#}$, then $\log m, \log m^{\prime} \in H$, so $g^{\#}(m, 0)=2^{g(\log m, 0)}=2^{g\left(\log m^{\prime}, 0\right)}=g^{\#}\left(m^{\prime}, 0\right)$.

4. Since $g$ is a scale, there exists $m_{0} \in H$ such that for all $m \geq m_{0}$, the function $s \mapsto g(m, s)$ is nonnegative and strictly increasing. For all $m \geq 2^{m_{0}}$, then, we have $\log m \geq m_{0}$, so the function $s \mapsto g^{\#}(m, s)=2^{g(\log m, s)}$ is nonnegative and strictly increasing.

5. Assume that $s^{\prime}>s \geq 0$. Since $g$ is a scale, there exists $m_{0} \in H$ such that for all $m \geq m_{0}$, $g(\log m, s) \geq 0$ and $\Delta(m) \geq 2$, where $\Delta(m)=g\left(\log m, s^{\prime}\right)-g(\log m, s)$. Then for all $m \geq m_{0}$ we have

$$
\begin{aligned}
g^{\#}\left(m, s^{\prime}\right)-g^{\#}(m, s) & =2^{g\left(\log m, s^{\prime}\right)}-2^{g(\log m, s)} \\
& =2^{g(\log m, s)}\left[2^{\Delta(m)}-1\right] \\
& \geq 2^{\Delta(m)}-1 \\
& \geq \Delta(m) \\
& =g\left(\log m, s^{\prime}\right)-g(\log m, s) .
\end{aligned}
$$

Since $\lim _{m \rightarrow \infty}\left[g\left(m, s^{\prime}\right)-g(m, s)\right]=\infty$, it follows immediately that $\lim _{m \rightarrow \infty}\left[g^{\#}\left(m, s^{\prime}\right)-g^{\#}(m, s)\right]=$ $\infty$.

Definition. If $g: H \times[0, \infty) \rightarrow \mathbb{R}$ is a scale, then the reflection of $g$ is the function $g^{R}: H \times$ $[0, \infty) \rightarrow \mathbb{R}$ defined by

$$
g^{R}(m, s)= \begin{cases}m+g(m, 0)-g(m, 1-s) & \text { if } 0 \leq s \leq 1 \\ g(m, s) & \text { if } s \geq 1\end{cases}
$$

Example 3.4. It is easy to verify that $g_{0}^{R}=g_{0}$ and that

$$
g_{1}^{R}(m, s)= \begin{cases}m+1-m^{1-s} & \text { if } 0 \leq s \leq 1 \\ m^{s} & \text { if } s \geq 1\end{cases}
$$

for all $m>0$ and $s \in \mathbb{R}$.

Lemma 3.5. If $g$ is a scale, then $g^{R}$ is a scale. 
Proof. Let $g: H \times[0, \infty) \rightarrow \mathbb{R}$ be a scale. It is clear that $g^{R}$ is continuous and has the same domain as $g$. Also, $g^{R}(m, 0)=g(m, 0)$ and $g^{R}(m, 1)=g(m, 1)$, so it suffices to prove that $g^{R}$ satisfies conditions 4 and 5 in the definition of a scale.

Let $m$ be large enough that $s \mapsto g(m, s)$ is nonnegative and strictly increasing, let $0 \leq s<s^{\prime} \leq 1$. It suffices to show that $0 \leq g^{R}(m, s)<g^{R}\left(m, s^{\prime}\right)$. For the first inequality, note that $1-s \leq 1$, so $g(m, 1-s) \leq g(m, 1)=m$, so $g^{R}(m, s)=m+g(m, 0)-g(m, 1-s) \geq g(m, 0) \geq 0$. For the second inequality, note that $1-s>1-s^{\prime}$, so $g(m, 1-s)>g\left(m, 1-s^{\prime}\right)$, so $g^{R}(m, s)<g^{R}\left(m, s^{\prime}\right)$. This confirms condition 4 .

Let $s^{\prime}>s \geq 0$. We have three cases.

(i) If $s \geq 1$, then

$$
\begin{aligned}
\lim _{m \rightarrow \infty}\left[g^{R}\left(m, s^{\prime}\right)-g^{R}(m, s)\right] & =\lim _{m \rightarrow \infty}\left[g\left(m, s^{\prime}\right)-g(m, s)\right] \\
& =\infty .
\end{aligned}
$$

(ii) If $s^{\prime} \leq 1$, then $1-s>1-s^{\prime} \geq 0$, so

$$
\begin{aligned}
\lim _{m \rightarrow \infty}\left[g^{R}\left(m, s^{\prime}\right)-g^{R}(m, s)\right] & =\lim _{m \rightarrow \infty}\left[g(m, 1-s)-g\left(m, 1-s^{\prime}\right)\right] \\
& =\infty .
\end{aligned}
$$

(iii) If $s<1$ and $s^{\prime}>1$, choose $m_{0} \in H$ such that $s \mapsto g(m, s)$ is nonnegative and strictly increasing for all $m \geq m_{0}$. Then for all $m \geq m_{0}$,

$$
\begin{aligned}
g^{R}\left(m, s^{\prime}\right)-g^{R}(m, s) & =g^{R}\left(m, s^{\prime}\right)-g^{R}(m, 1)+g^{R}(m, 1)-g^{R}(m, s) \\
& =g\left(m, s^{\prime}\right)-g(m, 1)+g^{R}(m, 1)-g^{R}(m, s) \\
& >g^{R}(m, 1)-g^{R}(m, s),
\end{aligned}
$$

so (ii) above (with $s^{\prime}=1$ ) tells us that $\lim _{m \rightarrow \infty}\left[g^{R}\left(m, s^{\prime}\right)-g^{R}(m, s)\right]=\infty$.

Notation. For each scale $g: H \times[0, \infty) \rightarrow \mathbb{R}$, we define the function $\Delta g: H \times[0, \infty) \rightarrow \mathbb{R}$ by

$$
\Delta g(m, s)=g(m+1, s)-g(m, s) .
$$

Note that $g$ is the usual finite difference operator, with the proviso that it is applied only to the first variable, $m$. For $l \in \mathbb{N}$, we also use the extended notation

$$
\Delta^{l} g(m, s)=g(m+l, s)-g(m, s) .
$$

The following definition is central to scaled dimension.

Definition. Let $g: H \times[0, \infty) \rightarrow \mathbb{R}$ be a scale, and let $s \in[0, \infty)$.

1. A $g$-scaled s-supergale (briefly, an $s^{(g)}$-supergale) is a function $d:\{0,1\}^{*} \longrightarrow[0, \infty)$ such that for all $w \in\{0,1\}^{*}$ with $|w| \in H$,

$$
d(w) \geq 2^{-\Delta g(|w|, s)}[d(w 0)+d(w 1)] .
$$

2. A g-scaled $s$-gale (briefly, an $s^{(g)}$-gale) is an $s^{(g)}$-supergale that satisfies (3.1) with equality for all $w \in\{0,1\}^{*}$ such that $|w| \in H$. 
3. An s-supergale is an $s^{\left(g_{0}\right)}$-supergale.

4. An $s$-gale is an $s^{\left(g_{0}\right)}$-gale.

5. A supermartingale is a 1-supergale.

6. A martingale is a 1-gale.

Remarks. 1. Martingales were introduced by Lévy [11] and named by Ville [22], who used them in early investigations of random sequences. Martingales were later used extensively by Schnorr $[18,19,20,21]$ in his investigations of random sequences and by Lutz $[13,16]$ in the development of resource-bounded measure. Gales were introduced by Lutz $[14,15]$ in the development of resource-bounded and constructive dimension. Scaled gales are introduced here in order to formulate scaled dimension.

2. Although the martingale condition is usually stated in the form

$$
d(w)=\frac{d(w 0)+d(w 1)}{2},
$$

this is a simplification of

$$
d(w) \mu(w)=d(w 0) \mu(w 0)+d(w 1) \mu(w 1),
$$

where $\mu(x)=2^{-|x|}$ is the measure (probability) of the cylinder $\mathbf{C}_{x}=\{A \in \mathbf{C} \mid x \sqsubseteq A\}$. Similarly, the $s$-gale condition

$$
d(w)=2^{-s}[d(w 0)+d(w 1)]
$$

of $[14,15]$ is a simplification of

$$
d(w) \mu(w)^{s}=d(w 0) \mu(w 0)^{s}+d(w 1) \mu(w 1)^{s},
$$

which is equivalent to

$$
d(w)=2^{-\Delta g_{0}(|w|, s)}[d(w 0)+d(w 1)] .
$$

In defining $s^{(g)}$-gales we have replaced the scale $g_{0}$ in (3.2) by an arbitrary scale $g$.

3. Condition (3.1) is only required to hold for strings $w$ that are long enough for $g(|w|, s)$ to be defined. In fact, several of the scales $g(m, s)$ used in this paper are not defined for small $m$. For such a scale $g$, an $s^{(g)}$-supergale must satisfy condition (3.1) for all but finitely many strings $w$, and this is sufficient for our development.

The following lemma is a generalization of Kraft's inequality.

Lemma 3.6. Let $g: H \times[0, \infty) \rightarrow \mathbb{R}$ be a scale, and let $s \in[0, \infty)$. If $d$ is an $s^{(g)}$-supergale and $B \subseteq\{0,1\}^{*}$ is a prefix set, then for all $w \in\{0,1\}^{*}$ with $|w| \in H$,

$$
\sum_{u \in B} 2^{-\Delta^{|u|} g(|w|, s)} d(w u) \leq d(w)
$$


Proof. Assume the hypothesis. We first use induction on $n$ to prove that for all $n \in \mathbb{N}$, the lemma holds for all prefix sets $B \subseteq\{0,1\}^{\leq n}$. For $n=0$, this is trivial. Assume that it holds for $n$, and let $A \subseteq\{0,1\}^{\leq n+1}$ be a prefix set. Let

$$
A^{\prime}=\left\{u \in\{0,1\}^{n} \mid u 0 \in A \text { or } u 1 \in A\right\},
$$

and let

$$
B=A_{\leq n} \cup A^{\prime} .
$$

Note that $B$ is a prefix set and $A_{\leq n} \cap A^{\prime}=\emptyset$ (because $A$ is a prefix set). Also, for all $w \in\{0,1\}^{*}$ with $|w| \in H$,

$$
\begin{aligned}
\sum_{u \in A=n+1} 2^{-\Delta^{|u|} g(|w|, s)} d(w u) & =2^{-\Delta^{n+1} g(|w|, s)} \sum_{u \in A_{=n+1}} d(w u) \\
& \leq 2^{-\Delta^{n+1} g(|w|, s)} \sum_{u \in A^{\prime}}[d(w u 0)+d(w u 1)] \\
& \leq 2^{-\Delta^{n+1} g(|w|, s)} \sum_{u \in A^{\prime}} 2^{\Delta g(|w u|, s)} d(w u) \\
& =2^{\Delta g(|w|+n, s)-\Delta^{n+1} g(|w|, s)} \sum_{u \in A^{\prime}} d(w u) \\
& =2^{-\Delta^{n} g(|w|, s)} \sum_{u \in A^{\prime}} d(w u) \\
& =\sum_{u \in A^{\prime}} 2^{-\Delta^{|u|} g(|w|, s)} d(w u) .
\end{aligned}
$$

Since $B \subseteq\{0,1\}^{\leq n}$, it follows by the induction hypothesis that for all $w \in\{0,1\}^{*}$ with $|w| \in H$, if we write

$$
\alpha(u)=2^{-\Delta^{|u|} g(|w|, s)} d(w u),
$$

then

$$
\begin{aligned}
\sum_{u \in A} \alpha(u) & =\sum_{u \in A_{\leq n}} \alpha(u)+\sum_{u \in A_{=n+1}} \alpha(u) \\
& \leq \sum_{u \in A_{\leq n}} \alpha(u)+\sum_{u \in A^{\prime}} \alpha(u) \\
& =\sum_{u \in B} \alpha(u) \\
& \leq d(w) .
\end{aligned}
$$

This completes the proof that for all $n \in \mathbb{N}$, the lemma holds for all prefix sets $B \subseteq\{0,1\} \leq n$.

To complete the proof of the lemma, let $B$ be an arbitrary prefix set. Then for all $w \in\{0,1\}^{*}$ with $|w| \in H$,

$$
\sum_{u \in B} \alpha(u)=\sup _{n \in \mathbb{N}} \sum_{u \in B \leq n} \alpha(u) \leq d(w) .
$$


Corollary 3.7. Let $g: H \times[0, \infty) \rightarrow \mathbb{R}$ be a scale, $s \in[0, \infty), 0<\alpha \in \mathbb{R}$, and $w \in\{0,1\}^{*}$ with $|w| \in H$. If $d$ is an $s^{(g)}$-supergale such that $d(w)>0$ and $B \subseteq\{0,1\}^{*}$ is a prefix set such that $d(w u) \geq \alpha 2^{\Delta^{|u|} g(|w|, s)-|u|} d(w)$ for all $u \in B$, then

$$
\sum_{u \in B} 2^{-|u|} \leq \frac{1}{\alpha}
$$

Proof. Assume the hypothesis. Then by Lemma 3.6,

$$
d(w) \geq \sum_{u \in B} 2^{-\Delta^{|u|} g(|w|, s)} d(w u) \geq \alpha d(w) \sum_{u \in B} 2^{-|u|},
$$

whence the corollary follows.

Corollary 3.8. Let $g: H \times[0, \infty) \rightarrow \mathbb{R}$ be a scale, let $s \in[0, \infty)$, and let $d$ be an $s^{(g)}$-supergale. Then for all $l \in \mathbb{N}, 0<\alpha \in \mathbb{R}$, and $w \in\{0,1\}^{*}$ with $|w| \in H$, there are fewer than $\frac{2^{l}}{\alpha}$ strings $u \in\{0,1\}^{l}$ for which

$$
\max _{v \sqsubseteq u} 2^{|v|-\Delta^{|v|} g(|w|, s)} d(w v)>\alpha d(w) .
$$

In particular, there is at least one string $u \in\{0,1\}^{l}$ such that $d(w v) \leq 2^{\Delta^{|v|} g(|w|, s)-|v|} d(w)$ for all $v \sqsubseteq u$.

Proof. Let $g, s, d, l, \alpha$, and $w$ be as given, and let

$$
A=\left\{u \in\{0,1\}^{l} \mid \max _{v \sqsubseteq u} 2^{|v|-\Delta^{|v|} g(|w|, s)} d(w v)>\alpha d(w)\right\} .
$$

Let $B$ be the set of all $v \in\{0,1\} \leq l$ such that $2^{|v|-\Delta^{|v|} g(|w|, s)} d(w v)>\alpha d(w)$ but $2^{\left|v^{\prime}\right|-\Delta^{\left|v^{\prime}\right|} g(|w|, s)} d\left(w v^{\prime}\right)$ $\leq \alpha d(w)$ for all $v^{\prime} \sqsubset v$. Then $B$ is a prefix set, and

$$
A=\left\{u \in\{0,1\}^{l} \mid(\exists v \sqsubseteq u) v \in B\right\}
$$

so $|A|=\sum_{v \in B} 2^{l-|v|}=2^{l} \sum_{v \in B} 2^{-|v|}$. Let $\alpha^{\prime}=\min _{v \in B} 2^{|v|-\Delta^{|v|} g(|w|, s)} \frac{d(w v)}{d(w)}$, and note that $\alpha<\alpha^{\prime}<$ $\infty$. Then $B$ is a prefix set such that $d(w v) \geq \alpha^{\prime} 2^{\Delta^{|v|} g(|w|, s)-|v|} d(w)$ for all $v \in B$, so Corollary 3.7 tells us that

$$
|A|=2^{l} \sum_{v \in B} 2^{-|v|} \leq \frac{2^{l}}{\alpha^{\prime}}<\frac{2^{l}}{\alpha} .
$$

This proves the main assertion of the corollary. The last sentence of the corollary follows by taking $\alpha=1$.

Corollary 3.9. Let $g: H \times[0, \infty) \rightarrow \mathbb{R}$ be a scale, let $s \in[0, \infty)$, and let $d$ be an $s^{(g)}$-supergale. Then for all $w, u \in\{0,1\}^{*}$ with $|w| \in H$,

$$
d(w u) \leq 2^{\Delta^{|u|} g(|w|, s)} d(w) .
$$

Proof. Let $g, s, d, w$, and $u$ be as given, and let $l=|u|$. Then Corollary 3.8 with $\alpha=2^{l}$ tells us that there are fewer than 1 , hence no strings $v \in\{0,1\}^{l}$ for which $d(w v)>2^{\Delta^{l} g(|w|, s)} d(w)$. Thus $d(w u) \leq 2^{\Delta^{|u|} g(|w|, s)} d(w)$. 
The following useful observations are now clear, as are the analogous observations for $s^{(g)}$ supergales.

Observation 3.10. Let $g: H \times[0, \infty) \rightarrow \mathbb{R}$ be a scale, let $m=\min (H \cap \mathbb{N})$, and let $s \in[0, \infty)$. For each $k \in \mathbb{N}$, let $d_{k}$ be an $s^{(g)}$-gale, and let $\alpha_{k} \in[0, \infty)$.

1. For each $n \in \mathbb{Z}^{+}, \sum_{k=0}^{n-1} \alpha_{k} d_{k}$ is an $s^{(g)}$-gale.

2. If $\sum_{k=0}^{\infty} \alpha_{k} d_{k}(w)<\infty$ for each $w \in\{0,1\}^{m}$, then $\sum_{k=0}^{\infty} \alpha_{k} d_{k}$ is an $s^{(g)}$-gale.

Observation 3.11. Let $g: H \times[0, \infty) \rightarrow \mathbb{R}$ be a scale, let $s, s^{\prime} \in[0, \infty)$, and let $d, d^{\prime}:\{0,1\}^{*} \rightarrow[0, \infty)$. If

$$
d(w) 2^{-g(|w|, s)}=d^{\prime}(w) 2^{-g\left(|w|, s^{\prime}\right)}
$$

for all $w \in\{0,1\}^{*}$ such that $|w| \in H$, then $d$ is an $s^{(g)}$-gale if and only if $d^{\prime}$ is an $s^{(g)}$-gale.

Definition. Let $g$ be a scale, let $s \in[0, \infty)$, and let $d$ be an $s^{(g)}$-supergale.

1. We say that $d$ succeeds on a language $A \in \mathbf{C}$ if $\limsup _{n \rightarrow \infty} d(A[0 \ldots n-1])=\infty$.

2. The success set of $d$ is $S^{\infty}[d]=\{A \in \mathbf{C} \mid d$ succeeds on $A\}$.

We now use scaled gales to define scaled dimension.

Notation. Let $g$ be a scale, and let $X \subseteq \mathbf{C}$.

1. $\mathcal{G}^{(g)}(X)$ is the set of all $s \in[0, \infty)$ such that there is an $s^{(g)}$-gale $d$ for which $X \subseteq S^{\infty}[d]$.

2. $\hat{\mathcal{G}}^{(g)}(X)$ is the set of all $s \in[0, \infty)$ such that there is an $s^{(g)}$-supergale $d$ for which $X \subseteq S^{\infty}[d]$.

Lemma 3.12. If $g$ is a scale, then for all $X \subseteq \mathbf{C}, \mathcal{G}^{(g)}(X)=\hat{\mathcal{G}}^{(g)}(X)$.

Proof. Let $s \in[0, \infty)$. Let $d$ be an $s^{(g)}$-supergale. We show that there is an $s^{(g)}$-gale $\tilde{d}$ such that $S^{\infty}[d] \subseteq S^{\infty}[\tilde{d}]$.

Define

$$
\begin{array}{lll}
\tilde{d}:\{0,1\}^{*} \longrightarrow[0, \infty) & \\
\tilde{d}(w) & =d(w) & \text { for }|w| \notin H \\
\tilde{d}(w 0) & =\frac{1}{2}\left[2^{-\Delta g(|w|, s)} \tilde{d}(w)+d(w 0)-d(w 1)\right] & \text { for }|w| \in H \\
\tilde{d}(w 1) & =\frac{1}{2}\left[2^{-\Delta g(|w|, s)} \tilde{d}(w)-d(w 0)+d(w 1)\right] & \text { for }|w| \in H
\end{array}
$$

Then $\tilde{d}$ is clearly an $s^{(g)}$-gale, and an easy induction shows that $\tilde{d}(w) \geq d(w)$ for all $w \in\{0,1\}^{*}$, whence $S^{\infty}[d] \subseteq S^{\infty}[\tilde{d}]$.

Recall the scale $g_{0}$ of Example 3.1. It was proven by Lutz [14] that the following definition is equivalent to the classical definition of Hausdorff dimension in $\mathbf{C}$.

Definition. The Hausdorff dimension of a set $X \subseteq \mathbf{C}$ is $\operatorname{dim}_{H}(X)=\inf \mathcal{G}^{\left(g_{0}\right)}(X)$.

This suggests the following rescaling of Hausdorff dimension in Cantor space.

Definition. If $g$ is a scale, then the $g$-scaled dimension of a set $X \subseteq \mathbf{C}$ is $\operatorname{dim}^{(g)}(X)=\inf \mathcal{G}^{(g)}(X)$. 
By Lemma 3.12, this definition would not be altered if we used $\hat{\mathcal{G}}^{(g)}(X)$ in place of $\mathcal{G}^{(g)}(X)$.

We now use resource-bounded scaled gales to develop scaled dimension in complexity classes. In the following, the resource bound $\Delta$ may be any one of the classes all, rec, $\mathrm{p}, \mathrm{p}_{2}, \mathrm{pspace}, \mathrm{p}_{2} \mathrm{space}_{\text {, }}$ etc., defined in section 2 .

Notation. If $g$ is a scale and $X \subseteq \mathbf{C}$, let $\mathcal{G}_{\Delta}^{(g)}(X)$ be the set of all $s \in[0, \infty)$ such that there is a $\Delta$-computable $s^{(g)}$-gale $d$ for which $X \subseteq S^{\infty}[d]$.

Definition. Let $g$ be a scale and $X \subseteq \mathbf{C}$.

1. The $g$-scaled $\Delta$-dimension of $X$ is $\operatorname{dim}_{\Delta}^{(g)}(X)=\inf \mathcal{G}_{\Delta}^{(g)}(X)$.

2. The $g$-scaled dimension of $X$ in $R(\Delta)$ is $\operatorname{dim}^{(g)}(X \mid R(\Delta))=\operatorname{dim}_{\Delta}^{(g)}(X \cap R(\Delta))$.

Note that $\operatorname{dim}_{\Delta}^{(g)}(X)$ and $\operatorname{dim}^{(g)}(X \mid R(\Delta))$ are defined for every scale $g$ and every set $X \subseteq \mathbf{C}$. Recalling the scale $g_{0}(m, s)=s m$, we write

$$
\begin{gathered}
\operatorname{dim}_{\Delta}(X)=\operatorname{dim}_{\Delta}^{\left(g_{0}\right)}(X), \\
\operatorname{dim}(X \mid R(\Delta))=\operatorname{dim}^{\left(g_{0}\right)}(X \mid R(\Delta))
\end{gathered}
$$

and note that these are exactly the resource-bounded dimensions defined by Lutz [14].

Observation 3.13. Let $g$ be a scale.

1. For all $X \subseteq Y \subseteq \mathbf{C}$,

$$
\operatorname{dim}_{\Delta}^{(g)}(X) \leq \operatorname{dim}_{\Delta}^{(g)}(Y)
$$

and

$$
\operatorname{dim}^{(g)}(X \mid R(\Delta)) \leq \operatorname{dim}^{(g)}(Y \mid R(\Delta))
$$

2. If $\Delta$ and $\Delta^{\prime}$ are resource bounds such that $\Delta \subseteq \Delta^{\prime}$, then for all $X \subseteq \mathbf{C}$,

$$
\operatorname{dim}_{\Delta^{\prime}}^{(g)}(X) \leq \operatorname{dim}_{\Delta}^{(g)}(X) .
$$

3. For all $X \subseteq \mathbf{C}, 0 \leq \operatorname{dim}^{(g)}(X \mid R(\Delta)) \leq \operatorname{dim}_{\Delta}^{(g)}(X)$.

4. For all $X \subseteq \mathbf{C}, \operatorname{dim}^{(g)}(X \mid \mathbf{C})=\operatorname{dim}_{\text {all }}^{(g)}(X)=\operatorname{dim}^{(g)}(X)$.

The following lemma relates resource-bounded scaled dimension to resource-bounded measure.

Lemma 3.14. If $g$ is a $\Delta$-computable scale, then for all $X \subseteq \mathbf{C}$,

$$
\operatorname{dim}_{\Delta}^{(g)}(X)<1 \Rightarrow \mu_{\Delta}(X)=0
$$

and

$$
\operatorname{dim}^{(g)}(X \mid R(\Delta))<1 \Rightarrow \mu(X \mid R(\Delta))=0 .
$$


Proof. It suffices to prove the first implication, since the second implication then follows immediately.

Assume that $\operatorname{dim}_{\Delta}^{(g)}(X)<1$, where $g$ is a $\Delta$-computable scale. Then there exists $s \in(0,1) \cap \mathbb{Q}$ and a $\Delta$-computable $s^{(g)}$-gale $d$ such that $X \subseteq S^{\infty}[d]$. Then the function $d^{\prime}:\{0,1\}^{*} \rightarrow[0, \infty)$ defined by

$$
d^{\prime}(w)=2^{|w|-g(|w|, s)} d(w)
$$

is $\Delta$-computable, and Observation 3.11 tells us that $d^{\prime}$ is a $1^{(g)}$-gale, i.e., a martingale. Since $g$ is a scale and $s<1$, we have $\lim _{m \rightarrow \infty}[m-g(m, s)]=\lim _{m \rightarrow \infty}[g(m, 1)-g(m, s)]=\infty$, so $X \subseteq S^{\infty}[d] \subseteq S^{\infty}\left[d^{\prime}\right]$. Thus $\mu_{\Delta}(X)=0$.

An important property of Hausdorff dimension is its stability [5], which is the fact that $\operatorname{dim}_{H}(X \cup$ $Y)$ is always the maximum of $\operatorname{dim}_{H}(X)$ and $\operatorname{dim}_{H}(Y)$. We now show that resource-bounded scaled dimensions also have this property.

Lemma 3.15. For every $\Delta$-computable scale $g$ and all sets $X, Y \subseteq \mathbf{C}$,

$$
\operatorname{dim}_{\Delta}^{(g)}(X \cup Y)=\max \left\{\operatorname{dim}_{\Delta}^{(g)}(X), \operatorname{dim}_{\Delta}^{(g)}(Y)\right\}
$$

and

$$
\operatorname{dim}^{(g)}(X \cup Y \mid R(\Delta))=\max \left\{\operatorname{dim}^{(g)}(X \mid R(\Delta)), \operatorname{dim}^{(g)}(Y \mid R(\Delta))\right\} .
$$

Proof. The second identity follows from the first, so by Observation 3.13 it suffices to show that

$$
\operatorname{dim}_{\Delta}^{(g)}(X \cup Y) \leq \max \left\{\operatorname{dim}_{\Delta}^{(g)}(X), \operatorname{dim}_{\Delta}^{(g)}(Y)\right\} .
$$

Choose an arbitrary $s>\max \left\{\operatorname{dim}_{\Delta}^{(g)}(X), \operatorname{dim}_{\Delta}^{(g)}(Y)\right\}$ such that $s$ is $\Delta$-computable. There exist $s_{1} \leq s$ and $\Delta$-computable $s_{1}^{(g)}$-gale $d_{1}$ such that $X \subseteq S^{\infty}\left[d_{1}\right]$, and $s_{2} \leq s$ and $\Delta$-computable $s_{2}^{(g)}$-gale $d_{2}$ such that $Y \subseteq S^{\infty}\left[d_{2}\right]$. Since $s$ is $\Delta$-computable, $d_{1}$ and $d_{2}$ are $\Delta$-computable $s^{(g)}$ supergales, and by the proof of Lemma $3.12 s \in \mathcal{G}_{\Delta}^{(g)}(X) \cap \mathcal{G}_{\Delta}^{(g)}(Y)$. So there exist $\Delta$-computable $s^{(g)}$-gales $d_{X}$ and $d_{Y}$ such that $X \subseteq S^{\infty}\left[d_{X}\right]$ and $Y \subseteq S^{\infty}\left[d_{Y}\right]$. Let $d=d_{X}+d_{Y}$. Then $d$ is clearly $\Delta$-computable, and $d$ is an $s^{(g)}$-gale by Observation 3.10. It is clear that $X \cup Y \subseteq S^{\infty}[d]$, whence $s \in \mathcal{G}_{\Delta}^{(g)}(X \cup Y)$. It follows that $\operatorname{dim}_{\Delta}^{(g)}(X \cup Y) \leq s$. Since $s$ is arbitrary here, we have shown that $\operatorname{dim}_{\Delta}^{(g)}(X \cup Y) \leq \max \left\{\operatorname{dim}_{\Delta}^{(g)}(X), \operatorname{dim}_{\Delta}^{(g)}(Y)\right\}$.

Hausdorff dimension is also countably stable [5], which means that the dimension of a countable union of sets is the supremum of the dimensions of the sets. The following definition and lemma show that resource-bounded scaled dimensions are " $\Delta$-stable" in the sense that they are stable relative to countable unions that are " $\Delta$-effective."

Definition. Let $g$ be a scale and let $X, X_{0}, X_{1}, X_{2}, \ldots \subseteq \mathbf{C}$.

1. $X$ is a $\Delta$-union of the $\Delta^{(g)}$-dimensioned sets $X_{0}, X_{1}, X_{2}, \ldots$ if $X=\bigcup_{k=0}^{\infty} X_{k}$ and for each rational $s>\sup _{k \in \mathbb{N}} \operatorname{dim}_{\Delta}^{(g)}\left(X_{k}\right)$ there is a function $d: \mathbb{N} \times\{0,1\}^{*} \rightarrow[0, \infty)$ with the following properties.

(i) $d$ is $\Delta$-computable.

(ii) For each $k \in \mathbb{N}$, if we write $d_{k}(w)=d(k, w)$, then the function $d_{k}$ is an $s^{(g)}$-gale.

(iii) For each $k \in \mathbb{N}, X_{k} \subseteq S^{\infty}\left[d_{k}\right]$. 
2. $X$ is a $\Delta$-union of the sets $X_{0}, X_{1}, X_{2}, \ldots{ }^{(g)}$-dimensioned in $R(\Delta)$ if $X=\bigcup_{k=0}^{\infty} X_{k}$ and $X \cap R(\Delta)$ is an $\Delta$-union of the $\Delta^{(g)}$-dimensioned sets $X_{0} \cap R(\Delta), X_{1} \cap R(\Delta), X_{2} \cap R(\Delta), \ldots$.

Lemma 3.16. Let $g$ be a $\Delta$-computable scale, and let $X, X_{0}, X_{1}, X_{2}, \ldots \subseteq \mathbf{C}$.

1. If $X$ is a $\Delta$-union of the $\Delta^{(g)}$-dimensioned sets $X_{0}, X_{1}, X_{2}, \ldots$, then

$$
\operatorname{dim}_{\Delta}^{(g)}(X)=\sup _{k \in \mathbb{N}} \operatorname{dim}_{\Delta}^{(g)}\left(X_{k}\right)
$$

2. If $X$ is a $\Delta$-union of the sets $X_{0}, X_{1}, X_{2}, \ldots{ }^{(g)}$-dimensioned in $R(\Delta)$, then

$$
\operatorname{dim}^{(g)}(X \mid R(\Delta))=\sup _{k \in \mathbb{N}} \operatorname{dim}^{(g)}\left(X_{k} \mid R(\Delta)\right) .
$$

Proof. We assume that $g$ is exactly $\Delta$-computable; the general proof is similar. It suffices to prove 1 , since 2 follows immediately from 1 . Assume the hypothesis of 1 , and let $s>\sup _{k \in \mathbb{N}} \operatorname{dim}_{\Delta}^{(g)}\left(X_{k}\right)$ be arbitrary with $s$ rational. By Observation 3.13, it suffices to show that $\operatorname{dim}_{\Delta}^{(g)}(X) \leq s$.

Since $X$ is a union of the $\Delta^{(g)}$-dimensioned sets $X_{0}, X_{1}, X_{2}, \ldots$, there is a $\Delta$-computable function $d: \mathbb{N} \times\{0,1\}^{*} \longrightarrow[0, \infty)$ such that each $d_{k}$ is an $s^{(g)}$-gale with $X_{k} \subseteq S^{\infty}\left[d_{k}\right]$. Without loss of generality (modifying $d$ if necessary), we can assume that each $d_{k}(w) \leq 1$ for each $w$ with $|w|=b$, $b=\min (H \cap \mathbb{N})$.

Let $\tilde{d}=\sum_{k=0}^{\infty} 2^{-k} d_{k}$. By Observation $3.10, \tilde{d}$ is an $s^{(g)}$-gale. Since $d$ is $\Delta$-computable, there is a function $\hat{d}: \mathbb{N} \times \mathbb{N} \times\{0,1\}^{*} \longrightarrow \mathbb{Q} \cap[0, \infty)$ such that $\hat{d} \in \Delta$ and for all $r, k \in \mathbb{N}$ and $w \in\{0,1\}^{*}$, $|\hat{d}(r, k, w)-d(k, w)| \leq 2^{-r}$. Define

$$
\hat{\tilde{\mathrm{d}}}: \mathbb{N} \times\{0,1\}^{*} \longrightarrow \mathbb{Q} \cap[0, \infty)
$$

For $w, s$ such that $g(|w|, s)-g(b, s) \geq 0$,

$$
\hat{\tilde{\mathrm{d}}}(r, w)=\sum_{k=0}^{r+g(|w|, s)-g(b, s)+1} 2^{-k} \hat{d}(r+2, k, w) .
$$

otherwise $(g(|w|, s)-g(b, s)<0)$,

$$
\hat{\tilde{d}}(r, w)=\sum_{k=0}^{r+1} 2^{-k} \hat{d}(r+2, k, w) .
$$

Then $\hat{\tilde{\mathrm{d}}} \in \Delta$. We look at $w \in\{0,1\}^{*}$ with $g(|w|, s)-g(b, s) \geq 0$, the other case is simpler. For all $r \in \mathbb{N}$,

$$
|\hat{\tilde{d}}(r, w)-\tilde{d}(w)| \leq|\tilde{d}(w)-c|+|c-\hat{\tilde{d}}(w)|,
$$

where $c=\sum_{k=0}^{r+g(|w|, s)-g(b, s)+1} 2^{-k} d_{k}(w)$. By Corollary 3.9,

$$
\begin{aligned}
& |\tilde{d}(w)-c|=\sum_{k=r+g(|w|, s)-g(b, s)+2}^{\infty} 2^{-k} d_{k}(w) \\
& \leq \sum_{k=r+g(|w|, s)-g(b, s)+2}^{\infty} 2^{-k} 2^{\Delta^{|w|-c} g(b, s)} \\
& =\sum_{k=r+g(|w|, s)-g(b, s)+2}^{\infty} 2^{g(|w|, s)-k-g(b, s)} \\
& =2^{-(r+1)} \text {. }
\end{aligned}
$$


Also,

$$
\begin{aligned}
|c-\hat{\tilde{\mathrm{d}}}(r, w)| & \leq \sum_{k=0}^{r+g(|w|, s)-g(b, s)+1} 2^{-k}|\hat{d}(r+2, k, w)-d(k, w)| \\
& \leq \sum_{k=0}^{\infty} 2^{-(k+r+2)} \\
& =2^{-(r+1)}
\end{aligned}
$$

It follows that for all $r \in \mathbb{N}$ and $w \in\{0,1\}^{*}$,

$$
|\hat{\tilde{d}}(r, w)-\tilde{d}(w)| \leq 2^{-r},
$$

whence $\hat{\tilde{d}}$ testifies that $\tilde{d}$ is $\Delta$-computable. It is clear that $X=\bigcup_{k=0}^{\infty} X_{k} \subseteq \bigcup_{k=0}^{\infty} S^{\infty}\left[d_{k}\right] \subseteq S^{\infty}[\tilde{d}]$, so it follows that $\operatorname{dim}_{\Delta}^{(g)}(X) \leq s$.

Definition. Let $d$ be an $s^{(g)}$-gale. The unitary success set of $d$ is

$$
S^{1}[d]=\{S \in \mathbf{C} \mid(\exists n) d(S[0 . . n-1]) \geq 1\} .
$$

A series $\sum_{n=0}^{\infty} a_{n}$ of nonnegative real numbers $a_{n}$ is $\Delta$-convergent if there is a function $m: \mathbb{N} \rightarrow$ $\mathbb{N}$ such that $m \in \Delta$ and

$$
\sum_{n=m(i)}^{\infty} a_{n} \leq 2^{-i}
$$

for all $i \in \mathbb{N}$. Such a function $m$ is called a modulus of the convergence. Adding a layer of uniformity, a sequence

$$
\sum_{k=0}^{\infty} a_{j, k} \quad(j=0,1,2, \ldots)
$$

of series of nonnegative real numbers is uniformly $\Delta$-convergent if there is a function $m: \mathbb{N}^{2} \rightarrow \mathbb{N}$ such that $m \in \Delta$ and, for all $j \in \mathbb{N}, m_{j}$ is a modulus of the convergence of the series $\sum_{k=0}^{\infty} a_{j, k}$.

We now further generalize the Borel-Cantelli lemma as was done for resource-bounded measure [13].

Lemma 3.17. Let $g: H \times[0, \infty) \rightarrow \mathbb{R}$ be a $\Delta$-computable scale, let $b=\min (H \cap \mathbb{N})$, and let $s \in[0, \infty)$. If $d: \mathbb{N}^{2} \times\{0,1\}^{*} \rightarrow[0, \infty)$ is a $\Delta$-computable function such that for each $j, k \in \mathbb{N} d_{j, k}$ is an $s^{(g)}$-gale, and such that for each $w$ with $|w|=b$ the series

$$
\sum_{k=0}^{\infty} d_{j, k}(w) \quad(j=0,1,2, \ldots)
$$

are uniformly $\Delta$-convergent, then

$$
\operatorname{dim}_{\Delta}^{(g)}\left(\bigcup_{j=0}^{\infty} \bigcap_{t=0}^{\infty} \bigcup_{k=t}^{\infty} S^{1}\left[d_{j, k}\right]\right) \leq s .
$$


Proof. Assume the hypothesis. Fix a function $m: \mathbb{N}^{2} \rightarrow \mathbb{N}$ testifying that the series (3.3) are uniformly $\Delta$-convergent for all $w$ with $|w|=b$. (The same $m$ can be valid for all $w$ because there are only finitely many $w$ with $|w|=b$.) Let $\hat{d}$ be a $\Delta$-computation of $d$.

Without loss of generality, assume that $m_{j}$ is nondecreasing and $m_{j}(n) \geq 2$ for all $j, n \in \mathbb{N}$. Define

$$
\begin{aligned}
S_{j, t} & =\bigcup_{k=t}^{\infty} S^{1}\left[d_{j, k}\right], \\
S_{j} & =\bigcap_{t=0}^{\infty} S_{j, t}, \text { and } \\
S & =\bigcup_{j=0}^{\infty} S_{j} .
\end{aligned}
$$

Our task is to prove that $\operatorname{dim}_{\Delta}^{(g)}(S) \leq s$.

Let $\epsilon>0$. Define $d^{\prime}: \mathbb{N} \times\{0,1\}^{*} \rightarrow[0, \infty)$ by

$$
d_{j}^{\prime}(w)=\sum_{k=0}^{\infty} d_{j, k}(w) \cdot 2^{g(|w|, s+\epsilon)-g(|w|, s)}
$$

for all $j \in \mathbb{N}$ and $w \in\{0,1\}^{*}$ with $|w| \in H$. For each $j \in \mathbb{N}, d_{j}^{\prime}$ is an $(s+\epsilon)^{(g)}$-gale by Observations 3.10 and 3.11. We will use the $\Delta$-union Lemma (3.16) to show that $d^{\prime}$ testifies that $\operatorname{dim}_{\Delta}^{(g)}(S) \leq s+\epsilon$.

To see that each $S_{j} \subseteq S^{\infty}\left[d_{j}^{\prime}\right]$, let $A \in S_{j}$. For each $t \in \mathbb{N}, A \in S_{j, t}$, so there exists a $k_{t} \geq m_{j}(t)$ and $l_{t} \in \mathbb{N}$ such that $d_{j, k_{t}}\left(A\left[0 . l_{t}-1\right]\right) \geq 1$. Then

$$
\begin{aligned}
d_{j}^{\prime}\left(A\left[0 . . l_{t}-1\right]\right) & \geq 2^{g\left(l_{t}, s+\epsilon\right)-g\left(l_{t}, s\right)} d_{j, k_{t}}\left(A\left[0 . . l_{t}-1\right]\right) \\
& \geq 2^{g\left(l_{t}, s+\epsilon\right)-g\left(l_{t}, s\right)} .
\end{aligned}
$$

By Corollary $3.9, d_{j, k_{t}}\left(A\left[0 . . l_{t}-1\right]\right) \leq 2^{-t} 2^{g\left(l_{t}, s\right)-g(b, s)}$, so $g\left(l_{t}, s\right) \geq t+g(b, s)$ and $l_{t}$ is unbounded. By the definition of scale, $2^{g\left(l_{t}, s+\epsilon\right)-g\left(l_{t}, s\right)}$ is unbounded as $t$ goes to infinity, so $A \in S^{\infty}\left[d_{j}^{\prime}\right]$.

To complete the proof, we need to show that $d^{\prime}$ is $\Delta$-computable. For each $j, r \in \mathbb{N}$ we define

$$
\hat{d}_{j, r}^{\prime}(w)=\sum_{k=0}^{m_{j}(r+1-g(|w|, s+\epsilon)-g(b, s))} \hat{d}_{j, k, r+k+2+g(|w|, s+\epsilon)-g(b, s)}(w) \cdot 2^{g(|w|, s+\epsilon)-g(|w|, s)} .
$$

Then $\hat{d}^{\prime} \in \Delta$ and for each $j, r \in \mathbb{N}$

$$
\begin{aligned}
\left|d_{j}^{\prime}(w)-\hat{d}_{j, r}^{\prime}(w)\right|= & 2^{g(|w|, s+\epsilon)-g(|w|, s)}\left(\sum_{k=m_{j}(r+1-g(|w|, s+\epsilon)-g(b, s))+1}^{\infty} d_{j, k}(w)\right. \\
& \left.+\left|\sum_{k=0}^{\mid m_{j}(r+1-g(|w|, s+\epsilon)-g(b, s))} d_{j, k}(w)-\hat{d}_{j, k, r+k+2+g(|w|, s+\epsilon)-g(|w|, s)}(w)\right|\right) \\
\leq & 2^{-(r+1)}+\sum_{k=0}^{m_{j}(r+1-g(|w|, s+\epsilon)-g(b, s))} 2^{-(r+k+2)} \\
\leq & 2^{-(r+1)}+2^{-(r+1)}=2^{-r} .
\end{aligned}
$$


We now show that singleton subsets of $R(\Delta)$ have scaled dimension 0 in $R(\Delta)$.

Lemma 3.18. If $g$ is a $\Delta$-computable scale, then for all $A \in R(\Delta)$,

$$
\operatorname{dim}^{(g)}(\{A\} \mid R(\Delta))=\operatorname{dim}_{\Delta}^{(g)}(\{A\})=0 .
$$

Proof. Assume the hypothesis, with $g: H \times[0, \infty) \rightarrow \mathbb{R}$, and let $s>0$ be rational. Let $m=$ $\min (H \cap \mathbb{N})$, and define

$$
\begin{gathered}
d:\{0,1\}^{*} \longrightarrow[0, \infty) \\
d(w)=\left\{\begin{array}{cc}
2^{g(m, s)} & \text { if } w \sqsubseteq A \text { and }|w|<m \\
2^{g(|w|, s)} & \text { if } w \sqsubseteq A \text { and }|w| \geq m \\
0 & \text { if } w \nsubseteq A .
\end{array}\right.
\end{gathered}
$$

The hypothesis implies that $d$ is $\Delta$-computable, and it is easily checked that $d$ is an $s^{(g)}$-gale. It is clear that $A \in S^{\infty}[d]$, whence $d$ testifies that $\operatorname{dim}_{\Delta}^{(g)}(\{A\}) \leq s$. Since $s$ is arbitrary here, it follows that $\operatorname{dim}_{\Delta}^{(g)}(\{A\})=0$.

Lemmas 3.15 and 3.18 immediately give the following.

Corollary 3.19. If $g$ is a $\Delta$-computable scale, then for all finite sets $X \subseteq R(\Delta)$,

$$
\operatorname{dim}^{(g)}(X \mid R(\Delta))=\operatorname{dim}_{\Delta}^{(g)}(X)=0 .
$$

In fact, Lemma 3.18 can be combined with $\Delta$-stability (Lemma 3.16) to show that all " $\Delta$ countable" subsets of $R(\Delta)$ have scaled dimension 0 in $R(\Delta)$. This implies, for example, that for all pspace-computable scales $g$ and all constants $c \in \mathbb{N}$,

$$
\operatorname{dim}^{(g)}\left(\operatorname{DSPACE}\left(2^{c n}\right) \mid \mathrm{ESPACE}\right)=0 .
$$

In contrast, even if $R(\Delta)$ is countable, $R(\Delta)$ does not have scaled dimension 0 in $R(\Delta)$. In fact we have the following.

Theorem 3.20. If $g$ is a $\Delta$-computable scale, then

$$
\operatorname{dim}^{(g)}(R(\Delta) \mid R(\Delta))=\operatorname{dim}_{\Delta}^{(g)}(R(\Delta))=\operatorname{dim}_{\Delta}^{(g)}(\mathbf{C})=1
$$

Proof. Let $g: H \times[0, \infty) \rightarrow \mathbb{R}$ be $\Delta$-computable. It is clear that

$$
\operatorname{dim}^{(g)}(R(\Delta) \mid R(\Delta))=\operatorname{dim}_{\Delta}^{(g)}(R(\Delta)) \leq \operatorname{dim}_{\Delta}^{(g)}(\mathbf{C}),
$$

so it suffices to prove that $\operatorname{dim}^{(g)}(R(\Delta) \mid R(\Delta)) \geq 1$ and $\operatorname{dim}_{\Delta}^{(g)}(\mathbf{C}) \leq 1$.

By the Measure Conservation Theorem $[13], \mu(R(\Delta) \mid R(\Delta))=1$, so by Lemma $3.14, \operatorname{dim}^{(g)}(R(\Delta) \mid$ $R(\Delta)) \geq 1$.

Let $s>1$ be rational, and define

$$
\begin{gathered}
d:\{0,1\}^{*} \longrightarrow[0, \infty) \\
d(w)= \begin{cases}2^{g\left(m_{0}, s\right)-m_{0}} & \text { if }|w|<m_{0} \\
2^{g(|w|, s)-|w|} & \text { if }|w| \geq m_{0},\end{cases}
\end{gathered}
$$

where $m_{0}=\min (H \cap \mathbb{N})$. Then $d$ is a $\Delta$-computable $s^{(g)}$-gale and $\lim _{m \rightarrow \infty}[g(m, s)-m]=\lim _{m \rightarrow \infty}[g(m, s)-$ $g(m, 1)]=\infty$ (because $g$ is a scale), so $\mathbf{C} \subseteq S^{\infty}[d]$. Thus $\operatorname{dim}_{\Delta}^{(g)}(\mathbf{C}) \leq s$. Since $s>1$ is arbitrary, this implies that $\operatorname{dim}_{\Delta}^{(g)}(\mathbf{C}) \leq 1$. 
We now define a particular family of scales that will be useful for studying the fractal structures of classes that arise naturally in computational complexity.

Definition. 1. For each $i \in \mathbb{N}$, define $a_{i}$ by the recurrence $a_{0}=-\infty, a_{i+1}=2^{a_{i}}$.

2. For each $i \in \mathbb{Z}$, define the $i^{\text {th }}$-order scale $g_{i}:\left(a_{|i|}, \infty\right) \times[0, \infty) \rightarrow \mathbb{R}$ by the following recursion.

(a) $g_{0}(m, s)=s m$.

(b) For $i \geq 0, g_{i+1}=g_{i}^{\#}$.

(c) For $i<0, g_{i}=g_{-i}^{R}$.

Note that each $g_{i}$ is a scale by Lemmas 3.3 and 3.5. It is easy to see that each $g_{i}$ is $\Delta$-computable.

Definition. Let $i \in \mathbb{Z}$ and $X \subseteq \mathbf{C}$.

1. The $i^{\text {th }}$-order dimension of $X$ is $\operatorname{dim}^{(i)}(X)=\operatorname{dim}^{\left(g_{i}\right)}(X)$.

2. The $i^{\text {th }}$-order $\Delta$-dimension of $X$ is $\operatorname{dim}_{\Delta}^{(i)}(X)=\operatorname{dim}_{\Delta}^{\left(g_{i}\right)}(X)$.

3. The $i^{\text {th }}$-order dimension of $X$ in $R(\Delta)$ is $\operatorname{dim}^{(i)}(X \mid R(\Delta))=\operatorname{dim}^{\left(g_{i}\right)}(X \mid R(\Delta))$.

In the spirit of the above definition, $s^{\left(g_{i}\right)}$-gales are now called $s^{(i)}$-gales, etc.

Intuitively, if $i<j$, then it is harder to succeed with an $s^{(j)}$-gale than with an $s^{(i)}$-gale, so $\operatorname{dim}^{(i)}(X) \leq \operatorname{dim}^{(j)}(X)$. We conclude this section by showing that even more is true.

Theorem 3.21. Let $i \in \mathbb{Z}$ and $X \subseteq \mathbf{C}$. If $\operatorname{dim}_{\Delta}^{(i+1)}(X)<1$, then $\operatorname{dim}_{\Delta}^{(i)}(X)=0$.

Proof. It can be proven by induction that for every $i \in \mathbb{Z}$, for arbitrary $s, \tilde{s}<1, \Delta g_{i+1}(m, s)=$ $o\left(\Delta g_{i}(m, \tilde{s})\right)$.

Assume the hypothesis. There exist an $s<1$ and a $\Delta$-computable $s^{(i+1)}$-gale $d$ such that $X \subseteq S^{\infty}[d]$. Take an arbitrary $\tilde{s}>0$. Since $\Delta g_{i+1}(m, s)=o\left(\Delta g_{i}(m, \tilde{s})\right)$, by changing only finitely many values we can transform $d$ into an $\tilde{s}^{(i)}$-supergale $\tilde{d}$ with $S^{\infty}[d]=S^{\infty}[\tilde{d}]$. It follows that $\operatorname{dim}_{\Delta}^{(i)}(X) \leq \tilde{s}$. Since $\tilde{s}$ was arbitrary, $\operatorname{dim}_{\Delta}^{(i)}(X)=0$.

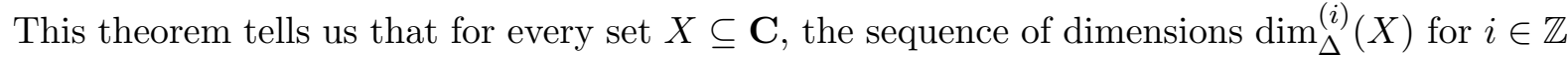
satisfies exactly one of the following three conditions.

(i) $\operatorname{dim}_{\Delta}^{(i)}(X)=0$ for all $i \in \mathbb{Z}$.

(ii) $\operatorname{dim}_{\Delta}^{(i)}(X)=1$ for all $i \in \mathbb{Z}$.

(iii) There exist $i^{*} \in \mathbb{Z}$ such that $\operatorname{dim}_{\Delta}^{(i)}(X)=0$ for all $i<i^{*}$ and $\operatorname{dim}_{\Delta}^{(i)}(X)=1$ for all $i>i^{*}$.

Intuitively, if condition (iii) holds and $0<\operatorname{dim}_{\Delta}^{\left(i^{*}\right)}(X)<1$, then $i^{*}$ is the "best" order at which to measure the $\Delta$-dimension of $X$ because $\operatorname{dim}_{\Delta}^{\left(i^{*}\right)}(X)$ provides more quantitative information about $X$ than is provided by $\operatorname{dim}_{\Delta}^{(i)}(X)$ for $i \neq i^{*}$. The following section provides some concrete examples of this phenomenon. 


\section{Nonuniform Complexity}

In this section we examine the scaled dimension of several nonuniform complexity classes in the complexity class ESPACE.

The circuit-size complexity of a language $A \subseteq\{0,1\}^{*}$ is the function $\mathrm{CS}_{A}: \mathbb{N} \rightarrow \mathbb{N}$, where $\mathrm{CS}_{A}(n)$ is the number of gates in the smallest $n$-input Boolean circuit that decides $A \cap\{0,1\}^{n}$. For each function $f: \mathbb{N} \rightarrow \mathbb{N}$, we define the circuit-size complexity classes

$$
\operatorname{SIZE}(f)=\left\{A \in \mathbf{C} \mid\left(\forall^{\infty} n\right) \mathrm{CS}_{A}(n) \leq f(n)\right\}
$$

and

$$
\operatorname{SIZE}_{\text {i.o. }}(f)=\left\{A \in \mathbf{C} \mid\left(\exists^{\infty} n\right) \mathrm{CS}_{A}(n) \leq f(n)\right\} .
$$

Given a machine $M$, a resource bound $t: \mathbb{N} \rightarrow \mathbb{N}$, a language $L \subseteq\{0,1\}^{*}$, and a natural number $n$, the $t$-space-bounded Kolmogorov complexity of $L_{=n}$ relative to $M$ is

$$
\operatorname{KS}_{M}^{t}\left(L_{=n}\right)=\min \left\{|\pi| \mid M(\pi, n)=\chi_{L_{=n}} \text { in } \leq t\left(2^{n}\right) \text { space }\right\},
$$

i.e., the length of the shortest program $\pi$ such that $M$, on input $(\pi, n)$, outputs the characteristic string of $L_{=n}$ and halts without using more than $t\left(2^{n}\right)$ workspace. Similarly the $t$-time-bounded Kolmogorov complexity of $L_{=n}$ relative to $M$ is

$$
\operatorname{KT}_{M}^{t}\left(L_{=n}\right)=\min \left\{|\pi| \mid M(\pi, n)=\chi_{L_{=n}} \text { in } \leq t\left(2^{n}\right) \text { time }\right\}
$$

Well-known simulation techniques show that there exists a machine $U$ which is optimal in the sense that for each machine $M$ there is a constant $c$ such that for all $t, L$ and $n$ we have

$$
\operatorname{KS}_{U}^{c t+c}\left(L_{=n}\right) \leq \operatorname{KS}_{M}^{t}\left(L_{=n}\right)+c
$$

and

$$
\mathrm{KT}_{U}^{c t \log t+c}\left(L_{=n}\right) \leq \mathrm{KT}_{M}^{t}\left(L_{=n}\right)+c .
$$

As usual, we fix such a universal machine and omit it from the notation.

For each resource bound $t: \mathbb{N} \rightarrow \mathbb{N}$ and function $f: \mathbb{N} \rightarrow \mathbb{N}$ we define the following complexity classes.

$$
\begin{aligned}
\mathrm{KS}^{t}(f) & =\left\{L \in \mathbf{C} \mid\left(\forall^{\infty} n\right) \mathrm{KS}^{t}\left(L_{=n}\right)<f(n)\right\} \\
\mathrm{KT}^{t}(f) & =\left\{L \in \mathbf{C} \mid\left(\forall^{\infty} n\right) \mathrm{KT}^{t}\left(L_{=n}\right)<f(n)\right\} \\
\mathrm{KS}_{\text {i.o. }}^{t}(f) & =\left\{L \in \mathbf{C} \mid\left(\exists^{\infty} n\right) \mathrm{KS}^{t}\left(L_{=n}\right)<f(n)\right\} \\
\mathrm{KT}_{\text {i.o. }}^{t}(f) & =\left\{L \in \mathbf{C} \mid\left(\exists^{\infty} n\right) \mathrm{KT}^{t}\left(L_{=n}\right)<f(n)\right\}
\end{aligned}
$$

Our first lemma provides inclusion relationships between some SIZE and KS classes defined using the scales.

Lemma 4.1. There exists a constant $c_{0} \in \mathbb{N}$ such that for all $i>0, \alpha \in[0,1]$, and $\epsilon>0$,

$$
\operatorname{SIZE}\left(g_{i}\left(2^{n}, \alpha\right)\right) \subseteq \operatorname{KS}^{c_{0} n+c_{0}}\left(g_{i}\left(2^{n}, \alpha+\epsilon\right)\right) .
$$


Proof. It was shown in [13] that there exists a polynomial $q_{0}$ and a constant $d$ such that for all $A \subseteq\{0,1\}^{*}$ and $n \in \mathbb{N}$,

$$
\mathrm{KS}^{q_{0}}\left(A_{=n}\right)<f_{A}(n)\left(d+\log f_{A}(n)\right),
$$

where $f_{A}(n)=\max \left\{\mathrm{CS}_{A}(n), n\right\}$. From that proof it is easy to see that $q_{0}$ may be taken as $c_{0} n+c_{0}$ for some $c_{0} \in \mathbb{N}$. Also, for $i>0$,

$$
g_{i}\left(2^{n}, \alpha\right)\left(d+\log g_{i}\left(2^{n}, \alpha\right)\right)=o\left(g_{i}\left(2^{n}, \alpha+\epsilon\right)\right) .
$$

The lemma follows using these facts.

The next two lemmas present positive-order dimension lower bounds for some SIZE classes.

Lemma 4.2. For all $i \geq 1$ and $\alpha \in(0,1]$, for all sufficiently large $n$ there are at least $2^{g_{i}\left(2^{n}, \alpha\right)}$ different sets $B \subseteq\{0,1\}^{n}$ that are decided by Boolean circuits of fewer than $g_{i}\left(2^{n}, \alpha\right)$ gates.

Proof. Let $m(n)=\left\lceil\log g_{i}\left(2^{n}, \alpha\right)\right\rceil$. For $n$ large enough, $m(n)<n$. Then there are $2^{2^{m(n)}} \geq 2^{g_{i}\left(2^{n}, \alpha\right)}$ different sets $C \subseteq\{0,1\}^{m(n)}$. Fix $\varepsilon>0$. For all sufficiently large $n$, Lupanov [12] has shown that each of these sets is decided by a circuit of at most $\frac{2^{m(n)}}{m(n)}(1+\varepsilon)$ gates. Now for sufficiently large $n$,

$$
\frac{2^{m(n)}}{m(n)}(1+\varepsilon) \leq \frac{2 g_{i}\left(2^{n}, \alpha\right)}{\log \left(g_{i}\left(2^{n}, \alpha\right)\right)}(1+\varepsilon)<g_{i}\left(2^{n}, \alpha\right) .
$$

Thus, for each $C \subseteq\{0,1\}^{m(n)}$, if we let $B_{C}=\{w x|w \in C| x \mid,=n-m(n)\}$, then $B_{C}$ is decided by a Boolean circuit of fewer than $g_{i}\left(2^{n}, \alpha\right)$ gates.

Lemma 4.3. For every $i \geq 1$, for every real $\alpha \in[0,1]$,

$$
\operatorname{dim}^{(i)}\left(\operatorname{SIZE}\left(g_{i}\left(2^{n}, \alpha\right)\right) \mid \operatorname{ESPACE}\right) \geq \alpha .
$$

Proof. This is clear if $\alpha=0$, so assume that $\alpha \in(0,1]$. Let $s, \alpha^{\prime} \in Q$ be such that $0<s<\alpha^{\prime}<\alpha$, and let $d$ be a pspace-computable $s^{(i)}$-gale. It suffices to show that $\operatorname{SIZE}\left(g_{i}\left(2^{n}, \alpha\right)\right) \cap \operatorname{ESPACE} \nsubseteq$ $S^{\infty}[d]$.

By Lemma 4.2 , there is an $N_{1}$ such that for all $n \geq N_{1}$, there are at least $2^{g_{i}\left(2^{n}, \alpha^{\prime}\right)}$ different sets $B \subseteq\{0,1\}^{n}$ that are decided by Boolean circuits of fewer than $g_{i}\left(2^{n}, \alpha^{\prime}\right)$ gates. By Corollary 3.8 , for all $w$ such that $|w|=2^{n}-1$, there are fewer than $2^{g_{i}\left(2^{n}, \alpha^{\prime}\right)}$ sets $B \subseteq\{0,1\}^{n}$ such that $d(w u)>2^{-g_{i}\left(2^{n}, \alpha^{\prime}\right)} 2^{\Delta^{2^{n}} g_{i}(|w|, s)} d(w)$, where $u$ is the characteristic string of $B$. Let $N_{2}$ be such that $\Delta^{2^{n}} g_{i}\left(2^{n}-1, s\right)-g_{i}\left(2^{n}, \alpha^{\prime}\right)<0$ for all $n \geq N_{2}$.

We now define a language $A$ inductively by lengths. Let $N=\max \left(N_{1}, N_{2}\right)$. We start with $A_{<N}=\emptyset$. Let $n \geq N$ and assume that $A_{<n}$ has been defined by characteristic string $w$. Let $u$ be the lexicographically first string of length $2^{n}$ such that $d(w u)<d(w)$ and the set with characteristic string $u$ can be decided by a circuit of less than $g_{i}\left(2^{n}, \alpha^{\prime}\right)$ gates. By the previous paragraph, $A$ is well-defined and $A \notin S^{\infty}[d]$. Since $d$ is pspace-computable, $A \in$ ESPACE, and by definition, $A \in \operatorname{SIZE}\left(g_{i}\left(2^{n}, \alpha^{\prime}\right)\right) \subseteq \operatorname{SIZE}\left(g_{i}\left(2^{n}, \alpha\right)\right)$.

We now give positive-order scaled dimension upper bounds for some KS classes defined using the scales.

Lemma 4.4. For all $i \geq 0$, for any polynomial $q$, and any $\alpha \in[0,1]$,

$$
\operatorname{dim}_{\text {pspace }}^{(i)}\left(\operatorname{KS}^{q}\left(g_{i}\left(2^{n}, \alpha\right)\right)\right) \leq \alpha .
$$


Proof. Let $q$ be a polynomial, let $\alpha \in(0,1)$, and let $s, \alpha^{\prime}$ be rational with $1>s>\alpha^{\prime}>\alpha$. Define $d: \mathbb{N} \times\{0,1\}^{*} \longrightarrow[0, \infty)$ inductively as follows. For $k \in \mathbb{N}$ with $2^{k} \geq a_{i}+2$, (recall that $\left.g_{i}:\left(a_{i}, \infty\right) \times[0, \infty) \rightarrow \mathbb{R}\right)$

(i) For $w \in\{0,1\}^{*}$ with $|w| \leq a_{i}+1$, let $d_{k}(w)=1$.

(ii) For $w \in\{0,1\}^{*}$ with $a_{i}+1 \leq|w|<2^{k}-1, b \in\{0,1\}$, let $d_{k}(w b)=2^{\Delta g_{i}(|w|, s)-1} d_{k}(w)$.

(iii) Assume that $d_{k}(w)$ has been defined, where $|w|=2^{n}-1$ for some $n \in \mathbb{N}, n \geq k$. For each $u$ with $0<|u| \leq 2^{n}$, define $d_{k}(w u)=2^{\Delta^{|u|} g_{i}(|w|, s)} \rho(u) d_{k}(w)$, where

$$
\rho(u)=\frac{\mid\left\{\pi|| \pi \mid<g_{i}\left(2^{n}, \alpha^{\prime}\right) \wedge u \sqsubseteq U(\pi, n) \text { in } \leq q\left(2^{n}\right) \text { space }\right\} \mid}{2^{g_{i}\left(2^{n}, \alpha^{\prime}\right)}-1} .
$$

It is easy to check that $d$ is pspace-computable and that for each $k, d_{k}$ is an $s^{(i)}$-gale. The definition of $d_{k}$ implies that if $|w|=2^{n}-1$ and $u$ is the characteristic string of a set $B \subseteq\{0,1\}^{n}$ with $\operatorname{KS}^{q}\left(B_{=n}\right)<g_{i}\left(2^{n}, \alpha\right)<g_{i}\left(2^{n}, \alpha^{\prime}\right)$, then for sufficiently large $n$,

$$
\begin{aligned}
d_{k}(w u) & \geq 2^{\Delta^{2^{n}} g_{i}(|w|, s)} \frac{1}{2^{g_{i}\left(2^{n}, \alpha^{\prime}\right)}-1} d_{k}(w) \\
& >2^{\Delta^{2^{n}} g_{i}(|w|, s)-g_{i}\left(2^{n}, \alpha^{\prime}\right)} d_{k}(w) \\
& =2^{g_{i}\left(2^{n+1}-1, s\right)-g_{i}\left(2^{n}-1, s\right)-g_{i}\left(2^{n}, \alpha^{\prime}\right)} d_{k}(w)
\end{aligned}
$$

Since $s>\alpha^{\prime}$,

$$
g_{i}\left(m, \alpha^{\prime}\right)=o\left(g_{i}(2 m-1, s)-g_{i}(m-1, s)\right)
$$

then for $n$ large enough, $d_{k}(w u) \geq 2 d_{k}(w)$. This implies that if

$$
Y_{k}=\left\{L \in \mathbf{C} \mid(\forall n \geq k) \operatorname{KS}^{q}\left(L_{=n}\right)<g_{i}\left(2^{n}, \alpha\right)\right\},
$$

$Y_{k} \subseteq S^{\infty}\left[d_{k}\right]$. Therefore $d$ witnesses that $\operatorname{KS}^{q}\left(g_{i}\left(2^{n}, \alpha\right)\right)$ is a pspace-union of the pspace ${ }^{(i)}$-dimensioned sets $Y_{0}, Y_{1}, \ldots$ Lemma 3.16 then yields

$$
\operatorname{dim}_{\text {pspace }}^{(i)}\left(\operatorname{KS}^{q}\left(g_{i}\left(2^{n}, \alpha\right)\right)\right) \leq \alpha .
$$

Now we are able to present exact scaled-dimension results for circuit-size complexity classes defined in terms of the positive scales. Note that in each case, we have obtained the "best" order at which to measure the dimension of the class.

Theorem 4.5. Let $i \geq 1$ and $\alpha \in[0,1]$. Then

$$
\operatorname{dim}^{(i)}\left(\operatorname{SIZE}\left(g_{i}\left(2^{n}, \alpha\right)\right) \mid \mathrm{ESPACE}\right)=\alpha .
$$

In particular,

$$
\operatorname{dim}^{(1)}\left(\operatorname{SIZE}\left(2^{\alpha n}\right) \mid \mathrm{ESPACE}\right)=\alpha
$$

and

$$
\operatorname{dim}^{(2)}\left(\operatorname{SIZE}\left(2^{n^{\alpha}}\right) \mid \operatorname{ESPACE}\right)=\alpha
$$


Proof. By Lemma 4.1 we have $\operatorname{SIZE}\left(g_{i}\left(2^{n}, \alpha\right)\right) \subseteq \mathrm{KS}^{c_{0} n+c_{0}}\left(2^{n}, \alpha+\epsilon\right)$ for all $\epsilon>0$. The theorem then follows from Lemmas 4.3 and 4.4.

At this point, we could use Lemmas 4.1 and 4.3 to give scaled dimension lower bounds for some KS classes defined using the positive scales. Also, proving an analogue of Lemma 4.1 for KT complexity will yield scaled dimension lower bounds for similar KT classes. However, taking a direct approach to these lower bounds yields slightly stronger results for KT complexity. In the next lemma we do this, and we also obtain scaled dimension lower bounds for all orders (not just the positive ones) at the same time.

Lemma 4.6. There exist constants $c_{1}, c_{2} \in \mathbb{N}$ such that for all $i \in \mathbb{Z}$ and $\alpha \in[0,1]$,

$$
\operatorname{dim}^{(i)}\left(\mathrm{KT}^{c_{1} n \log n+c_{1}}\left(g_{i}\left(2^{n}, \alpha\right)\right) \mid \mathrm{ESPACE}\right) \geq \alpha
$$

and

$$
\operatorname{dim}^{(i)}\left(\mathrm{KS}^{c_{2} n+c_{2}}\left(g_{i}\left(2^{n}, \alpha\right)\right) \mid \mathrm{ESPACE}\right) \geq \alpha .
$$

Proof. Let $s<\alpha$ be rational. Define $m(n)=\left\lceil g_{i}\left(2^{n}, s\right)\right\rceil$ for each $n \in \mathbb{N}$. For each $x \in\{0,1\}^{m(n)}$, let $B_{x} \subseteq\{0,1\}^{n}$ be the set with characteristic string $x 0^{2^{n}-m(n)}$. Let $M$ be a machine that on input $(x, n)$ outputs $x 0^{2^{n}-|x|}$. Then there are constants $c$ and $d$ such that for all $x \in\{0,1\}^{m(n)}$,

$$
\begin{aligned}
\mathrm{KT}_{U}^{c(d n) \log (d n)+c}\left(B_{x}\right) & \leq \mathrm{KT}_{M}^{d n}\left(B_{x}\right)+c \\
& \leq m(n)+c \\
& =\left\lceil g_{i}\left(2^{n}, s\right)\right\rceil+c .
\end{aligned}
$$

We let $c_{1}$ be such that $c d n \log (d n)+c \leq c_{1} n \log n+c_{1}$ for all $n$. For all sufficiently large $n$, $g_{i}\left(2^{n}, s\right)+c_{1}$ is bounded by $g_{i}\left(2^{n}, \alpha\right)$ since $s<\alpha$. Similarly, we obtain

$$
\mathrm{KS}_{U}^{c_{2} n+c_{2}}\left(B_{x}\right) \leq g_{i}\left(2^{n}, \alpha\right)
$$

for all $x \in\{0,1\}^{m(n)}$ and for some $c_{2}$.

Let $d$ be a pspace-computable $s^{(i)}$-gale. By Corollary 3.8, for all $w$ with $|w|=2^{n}-1$, there are fewer than $2^{g_{i}\left(2^{n}, s\right)}$ strings $u \in\{0,1\}^{2^{n}}$ such that $d(w u)>2^{-g_{i}\left(2^{n}, s\right)} 2^{\Delta^{2^{n}} g_{i}(|w|, s)} d(w)$. For all sufficiently large $n$, we have constructed at least $2^{g_{i}\left(2^{n}, s\right)}$ sets $B \subseteq\{0,1\}^{n}$ with $\mathrm{KT}^{c_{1} n \log n+c_{1}}(B) \leq$ $g_{i}\left(2^{n}, \alpha\right)$. As in the proof of Lemma 4.3 , we can define a language

$$
A \in\left[\mathrm{KT}^{c_{1} n \log n+c_{1}}\left(g_{i}\left(2^{n}, \alpha\right)\right) \cap \mathrm{ESPACE}\right]-S^{\infty}[d] .
$$

Analogously, we also obtain

$$
\operatorname{KS}^{c_{2} n+c_{2}}\left(g_{i}\left(2^{n}, \alpha\right)\right) \cap \operatorname{ESPACE} \nsubseteq \subseteq S^{\infty}[d] .
$$

Now we can state exact scaled dimension results for some KS and KT classes in the $0^{\text {th }}$ and positive-order scales.

Theorem 4.7. Let $i \geq 0, \alpha \in[0,1]$, and $t: \mathbb{N} \rightarrow \mathbb{N}$ be a polynomially-bounded function. Let $c_{1}$ and $c_{2}$ be as in Lemma 4.6. If $t(n) \geq c_{1} n \log n+c_{1}$ almost everywhere, then

$$
\operatorname{dim}^{(i)}\left(\operatorname{KT}^{t}\left(g_{i}\left(2^{n}, \alpha\right)\right) \mid \mathrm{ESPACE}\right)=\alpha,
$$


and if $t(n) \geq c_{2} n+c_{2}$ almost everywhere, then

$$
\operatorname{dim}^{(i)}\left(\operatorname{KS}^{t}\left(g_{i}\left(2^{n}, \alpha\right)\right) \mid \mathrm{ESPACE}\right)=\alpha .
$$

In particular, for any polynomial $q(n) \geq n^{2}$,

$$
\operatorname{dim}^{(1)}\left(\mathrm{KT}^{q}\left(2^{\alpha n}\right) \mid \mathrm{ESPACE}\right)=\operatorname{dim}^{(1)}\left(\mathrm{KS}^{q}\left(2^{\alpha n}\right) \mid \mathrm{ESPACE}\right)=\alpha,
$$

and

$$
\operatorname{dim}^{(2)}\left(\mathrm{KT}^{q}\left(2^{n^{\alpha}}\right) \mid \mathrm{ESPACE}\right)=\operatorname{dim}^{(2)}\left(\mathrm{KS}^{q}\left(2^{n^{\alpha}}\right) \mid \mathrm{ESPACE}\right)=\alpha .
$$

Proof. This follows immediately from Lemmas 4.4 and 4.6.

Now we give an upper bound on the scaled dimension of some KS classes for the negative scales. In the negative orders, we are able to work with classes of the infinitely-often type.

Lemma 4.8. Let $i \leq-1, q$ be a polynomial, and $\alpha \in[0,1]$. Then

$$
\operatorname{dim}_{\text {pspace }}^{(i)}\left(\operatorname{KS}_{\text {i.o. }}^{q}\left(g_{i}\left(2^{n}, \alpha\right)\right)\right) \leq \alpha .
$$

Proof. Let $q$ be a polynomial, let $\alpha \in(0,1)$, and let $s, \alpha^{\prime}$ be rational such that $1>s>\alpha^{\prime}>\alpha$. Define for each $n \in \mathbb{N}$ a function $d_{n}:\{0,1\}^{*} \longrightarrow[0, \infty)$ inductively as follows. For $n \in \mathbb{N}$ with $2^{n} \geq a_{|i|}+2$,

(i) For $w \in\{0,1\}^{*}$ with $|w| \leq a_{|i|}+1$, let $d_{n}(w)=2^{-g_{|i|}\left(2^{n}, 1-s\right)}$.

(ii) For $w \in\{0,1\}^{*}$ with $a_{|i|}+1 \leq|w|<2^{n}-1, b \in\{0,1\}$, let $d_{n}(w b)=2^{\Delta g_{i}(|w|, s)-1} d_{n}(w)$.

(iii) Assume that $d_{n}(w)$ has been defined, where $|w|=2^{n}-1$. For each $u$ with $0<|u| \leq 2^{n}$, define $d_{n}(w u)=2^{\Delta^{|u|} g_{i}(|w|, s)} \rho(u) d_{n}(w)$, where

$$
\rho(u)=\frac{\mid\left\{\pi|| \pi \mid<g_{i}\left(2^{n}, \alpha^{\prime}\right) \wedge u \sqsubseteq U(\pi, n) \text { in } \leq q\left(2^{n}\right) \text { space }\right\} \mid}{2^{g_{i}\left(2^{n}, \alpha^{\prime}\right)}-1} .
$$

(iv) For $w \in\{0,1\}^{*}$ with $|w| \geq 2^{n+1}-1, b \in\{0,1\}$, let $d_{n}(w b)=2^{\Delta g_{i}(|w|, s)-1} d_{n}(w)$

It is easy to check that for each $n, d_{n}$ is a pspace-computable $s^{(i)}$-gale. The definition of $d_{n}$ implies that if $|w|=2^{n}-1$ and $u$ is the characteristic string of a set $B \subseteq\{0,1\}^{n}$ with $\operatorname{KS}^{q}\left(B_{=n}\right)<$ $g_{i}\left(2^{n}, \alpha\right)<g_{i}\left(2^{n}, \alpha^{\prime}\right)$, then for sufficiently large $n$,

$$
\begin{aligned}
& d_{n}(w u) \geq 2^{\Delta^{2^{n}} g_{i}(|w|, s)} \frac{1}{2^{g_{i}\left(2^{n}, \alpha^{\prime}\right)}-1} d_{n}(w) \\
& >2^{\Delta^{2^{n}} g_{i}(|w|, s)-g_{i}\left(2^{n}, \alpha^{\prime}\right)} d_{n}(w) \\
& =2^{\Delta^{2^{n}} g_{i}(|w|, s)-g_{i}\left(2^{n}, \alpha^{\prime}\right)} 2^{\Delta^{|w|-a_{|i|}-1}} g_{i}\left(a_{|i|}+1, s\right)-|w|+a_{|i|}+1-g_{|i|}\left(2^{n}, 1-s\right) \\
& =2^{g_{i}\left(2^{n+1}-1, s\right)-g_{i}\left(a_{|i|}+1, s\right)-g_{i}\left(2^{n}, \alpha^{\prime}\right)-|w|+a_{|i|}+1-g_{|i|}\left(2^{n}, 1-s\right)} \\
& =2^{g_{|i|}\left(2^{n}, 1-\alpha^{\prime}\right)-g_{|i|}\left(2^{n+1}-1,1-s\right)-g_{|i|}\left(2^{n}, 1-s\right)+g_{|i|}\left(a_{|i|}+1,1-s\right)-g_{|i|}\left(2^{n}, 0\right)}
\end{aligned}
$$

Since $s>\alpha^{\prime}$,

$$
g_{|i|}(2 m-1,1-s)+g_{|i|}(m, 1-s)=o\left(g_{|i|}\left(m, 1-\alpha^{\prime}\right)\right.
$$


Then for $n$ large enough, $d_{n}(w u) \geq 1$. This implies that if

$$
Y_{n}=\left\{L \in \mathbf{C} \mid \operatorname{KS}^{q}\left(L_{=n}\right)<g_{i}\left(2^{n}, \alpha\right)\right\},
$$

then $Y_{n} \subseteq S^{1}\left[d_{n}\right]$. Since for each $w$ with $|w|=a_{|i|}+1, \sum_{n=0}^{\infty} d_{n}(w)$ is pspace-convergent, by Lemma 3.17 it holds that $\operatorname{dim}_{\text {pspace }}^{(i)}\left(\operatorname{KS}_{\text {i.o }}^{q}\left(2^{g_{i}\left(2^{n}, \alpha\right)}\right)\right) \leq \alpha$.

Our final theorem is an exact scaled dimension result analogous to Theorem 4.7 for the negative scales. Here the dimension is invariant if we change the type of the class from almost-everywhere to infinitely-often.

Theorem 4.9. Let $i \leq-1, \alpha \in[0,1]$, and $t: \mathbb{N} \rightarrow \mathbb{N}$ be a polynomially-bounded function. Let $c_{1}$ and $c_{2}$ be as in Lemma 4.6. If $t(n) \geq c_{1} n \log n+c_{1}$ almost everywhere, then

$$
\operatorname{dim}^{(i)}\left(\operatorname{KT}^{t}\left(g_{i}\left(2^{n}, \alpha\right)\right) \mid \mathrm{ESPACE}\right)=\operatorname{dim}^{(i)}\left(\mathrm{KT}_{\mathrm{i} . \mathrm{o} .}^{t}\left(g_{i}\left(2^{n}, \alpha\right)\right) \mid \mathrm{ESPACE}\right)=\alpha,
$$

and if $t(n) \geq c_{2} n+c_{2}$ almost everywhere,

$$
\operatorname{dim}^{(i)}\left(\mathrm{KS}^{t}\left(g_{i}\left(2^{n}, \alpha\right)\right) \mid \mathrm{ESPACE}\right)=\operatorname{dim}^{(i)}\left(\mathrm{KS}_{\mathrm{i} . \mathrm{o}}^{t}\left(g_{i}\left(2^{n}, \alpha\right)\right) \mid \mathrm{ESPACE}\right)=\alpha .
$$

In particular, for any polynomial $q(n) \geq n^{2}$,

$$
\left.\left.\operatorname{dim}^{(-1)}\left(\operatorname{KT}^{q}\left(2^{n}\left(1-2^{-\alpha n}\right)\right)\right) \mid \operatorname{ESPACE}\right)=\operatorname{dim}^{(-1)}\left(\operatorname{KS}^{q}\left(2^{n}\left(1-2^{-\alpha n}\right)\right)\right) \mid \operatorname{ESPACE}\right)=\alpha .
$$

Proof. This follows from Lemmas 4.6 and 4.8 .

\section{Acknowledgements}

We thank two anonymous referees and María López for a careful review.

\section{References}

[1] K. Ambos-Spies, W. Merkle, J. Reimann, F. Stephan, Hausdorff dimension in exponential time, in: Proceedings of the 16th IEEE Conference on Computational Complexity, 2001, pp. $210-217$.

[2] K. B. Athreya, J. M. Hitchcock, J. H. Lutz, E. Mayordomo, Effective strong dimension, algorithmic information, and computational complexity, Tech. Rep. cs.CC/0211025, Computing Research Repository (2002).

[3] R. Beigel, L. Fortnow, F. Stephan, Infinitely-often autoreducible sets, in: Proceedings of the 14th Annual International Symposium on Algorithms and Computation, 2003. To appear.

[4] H. Eggleston, The fractional dimension of a set defined by decimal properties, Q. J. Math. 20 (1949) 31-36.

[5] K. Falconer, Fractal Geometry: Mathematical Foundations and Applications, John Wiley \& Sons, 1990.

[6] L. Fortnow, J. H. Lutz, Prediction and dimension, J. Comput. Syst. Sci. To appear. 
[7] F. Hausdorff, Dimension und äusseres Mass, Math. Ann. 79 (1919) 157-179.

[8] J. M. Hitchcock, Fractal dimension and logarithmic loss unpredictability, Theoretical Comput. Sci. 304 (1-3) (2003) 431-441.

[9] J. M. Hitchcock, MAX3SAT is exponentially hard to approximate if NP has positive dimension, Theoretical Comput. Sci. 289 (1) (2002) 861-869.

[10] D. W. Juedes, J. H. Lutz, Completeness and weak completeness under polynomial-size circuits, Inform. and Comp. 125 (1) (1996) 13-31.

[11] P. Lévy, Théorie de l'Addition des Variables Aleatoires, Gauthier-Villars, 1937 (second edition 1954).

[12] O. B. Lupanov, On the synthesis of contact networks, Dokl. Akad. Nauk SSSR 119 (1958) pp. $23-26$.

[13] J. H. Lutz, Almost everywhere high nonuniform complexity, J. Comput. Syst. Sci. 44 (2) (1992) $220-258$.

[14] J. H. Lutz, Dimension in complexity classes, SIAM J. Comput. 32 (5) (2003) 1236-1250. Preliminary version appeared in Proceedings of the Fifteenth Annual IEEE Conference on Computational Complexity, pages 158-169, 2000.

[15] J. H. Lutz, The dimensions of individual strings and sequences, Inform. and Comp. To appear.

[16] J. H. Lutz, Resource-bounded measure, in: Proceedings of the 13th IEEE Conference on Computational Complexity, 1998, pp. 236-248.

[17] C. A. Rogers, Hausdorff Measures, Cambridge University Press, 1998, originally published in 1970.

[18] C. P. Schnorr, Klassifikation der Zufallsgesetze nach Komplexität und Ordnung, Z. Wahrscheinlichkeitstheorie verw. Geb. 16 (1970) 1-21.

[19] C. P. Schnorr, A unified approach to the definition of random sequences, Math. Systems Theory 5 (1971) 246-258.

[20] C. P. Schnorr, Zufälligkeit und Wahrscheinlichkeit, Lecture Notes in Math. 218.

[21] C. P. Schnorr, Process complexity and effective random tests, J. Comput. Syst. Sci. 7 (1973) $376-388$.

[22] J. Ville, Étude Critique de la Notion de Collectif, Gauthier-Villars, Paris, 1939. 